\title{
Badehauben-großes mikrozystisches Adnexkarzinom in Kollision mit einem Bowenkarzinom nach photodynamischer Therapie
}

\author{
Bathing Cap-Like Microcystic Adnexal Carcinoma Associated with a Bowen \\ Carcinoma after Photodynamic Therapy
}

Autoren

Institut
C. Schiekofer, C. S. L. Müller, S. Psaier, T. Vogt, K. Rass

Klinik für Dermatologie, Venerologie und Allergologie, Universitätsklinikum des Saarlandes, Homburg/Saar
Bibliografie

DOI http://dx.doi.org/

10.1055/s-0030-1256129

Akt Dermatol 2011; 37:

27-30 @ Georg Thieme

Verlag KG Stuttgart · New York

ISSN 0340-2541

Korrespondenzadresse

Claudia Schiekofer

Klinik für Dermatologie, Venerologie und Allergologie Universitätsklinikum des Saarlandes

Kirrbergerstr. 1 - Gebäude 18 66421 Homburg

claudia.schiekofer@uks.eu

\section{Zusammenfassung \\ $\nabla$}

Das mikrozystische Adnexkarzinom (MAK) ist ein seltener, häufig über Jahre langsam wachsender und flächig infiltrierender Tumor der Schweißdrüsen, der histologisch durch oberflächliche Epithelverbände mit zentralen Hornzysten sowie syringoiden Epithelverbänden mit perineuraler Infiltration in den tieferen Abschnitten gekennzeichnet ist. Wir berichten über einen 71-jährigen Patienten, der sich aufgrund einer Feldkanzerisierung im Bereich des Capillitiums vorstellte. 7 Jahre zuvor war eine photodynamische Therapie (PDT) erfolgt. Es wurde eine erneute PDT sowie die Exzision einer „Plattenepithelkarzinom“-verdächtigen Läsion am Hinterkopf durchgeführt. Histologisch zeigte sich hierbei ein Bowenkarzinom in Assoziation mit einem dermalen MAK. Im

\section{Einleitung \\ $\nabla$}

Das mikrozystische Adnexkarzinom (MAK), erstmals 1982 von Goldstein beschrieben, ist ein seltener maligner Hauttumor mit ekkriner Differenzierung [1]. Es wird u.a. auch als sklerosierendes Schweißdrüsen-Karzinom oder malignes Syringom bezeichnet [2]. Charakteristisch ist ein langsames, jedoch typischerweise weit über das klinisch vermutete Ausmaß hinausgehendes diffuses Wachstum und das Auftreten im Kopf-HalsBereich $[3,4]$. Histologisch zeichnet sich das MAK durch einen zweischichtigen Aufbau mit oberflächlichen Keratinzysten und tiefer gelegenen syringoiden Zellverbänden mit umgebendem hyalinen Stroma aus [5]. Von kausaler Bedeutung könnte eine chronische UV-Exposition sein [6]; über das Auftreten von MAK in bestrahlten Hautgebieten liegen Fallberichte vor [6].
Rahmen der mikrografisch kontrollierten Chirurgie ergab sich nach insgesamt 9 Exzisionen ein Defekt, der das gesamte Capillitium einnahm. Eine R0-Situation war ohne gesichtsentstellende Operation nicht erreichbar. Der Defekt wurde daher mittels Spalthauttransplantat verschlossen und es wurde eine Bestrahlung angeschlossen. 6 Monate nach Abschluss der Radiatio besteht histologisch kontrollierte Rezidivfreiheit. Der vorliegende Fall zeigt, dass das MAK klinisch inapparent sein kann und dennoch eine extreme Ausdehnung aufweisen kann. Über die Ätiopathogenese des MAK ist angesichts der Seltenheit des Tumors wenig bekannt. Eine Induktion durch UVund ionisierende Strahlung wird diskutiert. Ob ein kausaler Zusammenhang mit der vorausgegangenen PDT besteht, bleibt spekulativ.

\section{Kasuistik \\ $\nabla$}

Wir berichten hier über einen 71-jährigen männlichen Patienten, der sich aufgrund einer „Feldkanzerisierung“ im Bereich des Capillitiums im September 2009 zur photodynamischen Therapie (PDT) in unserer Klinik vorstellte. Bereits sieben Jahre zuvor war eine PDT des Capillitiums sowie die Exzision eines Bowenkarzinoms frontal und multipler weiterer Carcinomata in situ im Kopfbereich erfolgt. Aufgrund seiner beruflichen Tätigkeit als Bauarbeiter war der Patient viele Jahre lang einer starken Sonnenexposition ausgesetzt. An internistischen Erkrankungen wurde über eine Arrhythmia absoluta bei Vorhofflimmern, eine arterielle Hypertonie und eine Heparin-induzierte Thrombopenie Typ I berichtet.

Bei der klinischen Aufnahmeuntersuchung fielen neben multiplen rauen, teils erythematösen Makulae am Capillitium parieto-occipital infiltrierte, bräunlich krustig belegte, nicht schmerzhafte Plaques auf $(\bullet$ Abb. 1). 


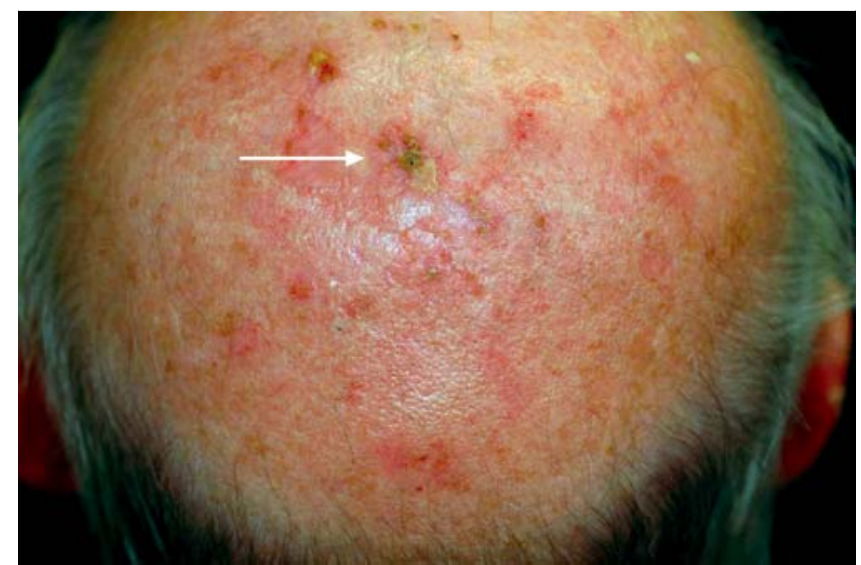

Abb. 1 Aufnahmebefund mit klinischer Darstellung einer Feldkanzerisierung des Capillitiums sowie einer stärker infiltrierten, bräunlich krustig belegten Plaque parieto-occipital (Pfeil), die histologisch einem Bowenkarzinom entsprach.

Im Rahmen der in Intubationsnarkose erneut durchgeführten PDT wurde die infiltrierte Hautveränderung parieto-occipital unter der klinischen Verdachtsdiagnose eines Plattenepithelkarzinoms exzidiert.

Histologisch zeigten sich eine akanthotisch verbreiterte Epidermis mit komplettem Verlust der physiologischen Schichtung und zahlreichen Zell- und Kernpolymorphien sowie Pyknosen im Sinne eines Morbus Bowen bzw. initialen Bowenkarzinoms. In der papillären Dermis fielen Keratinzysten auf und kleinere, teils syringoide Zellverbände mit blassem Zytoplasma und monomorphen Zellkernen ( $\bullet$ Abb. 2 a).

In der retikulären Dermis fanden sich ebenfalls kleine, teils tubuläre Epithelverbände in fibrosiertem Stroma mit vereinzeltem

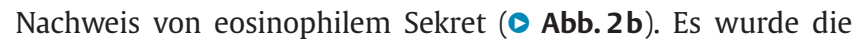
Diagnose eines mikrozystischen Adnexkarzinoms (MAK) in Kollision mit einem Bowenkarzinom in chronisch lichtgeschädigter Haut gestellt - beides nach der ersten Exzision non in sano.

Nach Vorlage der histologischen Befunde erfolgte zunächst der Versuch einer operativen Sanierung des MAK in einem mehrzeitigen Vorgehen mit histografischen Schnittrandkontrollen. Nach insgesamt 9 Exzisionen mit dem Ergebnis einer kompletten Entfernung des Capillitiums mit einer Defektgröße von ca. $25 \mathrm{~cm} \times$ $20 \mathrm{~cm}$ ( $\bullet$ Abb.3) bestand zwar eine R0-Situation bzgl. des Bowenkarzinoms, aber immer noch eine R1-Situation des MAK in frontotemporaler Richtung.

Das Erreichen einer vollständigen Exzision des MAK wurde zunehmend unwahrscheinlich und wäre allenfalls nur durch eine gesichtsentstellende weitere Operation zu erreichen gewesen, $d$. h. Entfernung von Stirnhaut und Augenbrauen. Nach ausführli-

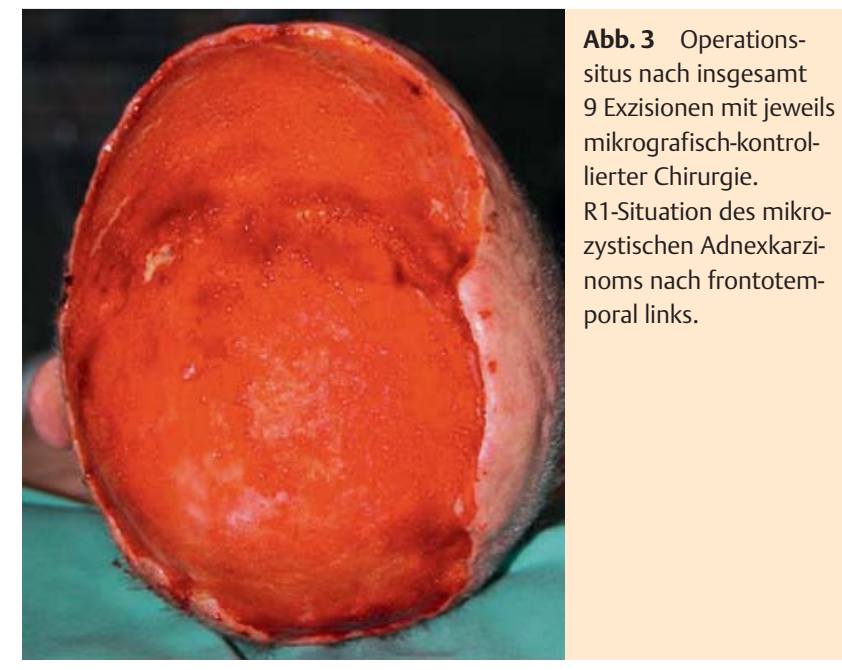

cher Erörterung des Befundes mit dem Patienten und nach Einholung eines strahlentherapeutischen Konsils entschieden wir uns bei relativer Inoperabilität des MAK für eine Radiatio nach vorherigem Defektverschluss.

Im Januar 2010 erfolgte der Defektverschluss nach ausreichender Wundkonditionierung mit Polyvinylalkohol-Schwamm mittels Spalthauttransplantation von beiden ventralen Oberschenkeln und nachfolgender V.A.C.-Therapie für eine Woche. Nach Abschluss der Wundheilung ( Abb.4au.b) erfolgten zunächst Ausbreitungsbiopsien nach frontotemporal links in einem Abstand von $2-3 \mathrm{~cm}$ zum ehemaligen Wundrand. Histologisch waren hierbei keine Anteile des MAK mehr nachweisbar.

Von 02 / 2010 bis 04/2010 wurde das perioperative Areal mit einem Abstand von $3 \mathrm{~cm}$ um das Transplantat zirkulär bestrahlt. Die Strahlendosis betrug insgesamt 66 Gy à 2 Gy ED, wobei nach konventioneller Bestrahlungsplanung eine Stehfeldtechnik mit insgesamt 3 Feldern mit Elektronen der Grenzenergie $8 \mathrm{MeV}$ zur Anwendung kam.

Da kontroverse Daten zur Effektivität der Strahlentherapie beim MAK vorliegen, wurden mit dem Patienten engmaschige klinische Kontrollen sowie regelmäßige Re-Biopsien vereinbart. Bei Wiedervorstellung im September 2010 erfolgten die ersten bioptischen Kontrollen, die keinen Anhalt für ein Rezidiv des MAK zeigten.

\section{Diskussion \\ $\nabla$}

Das MAK ist ein seltener, häufig über Jahre langsam und typischerweise weit über das klinisch vermutete Ausmaß hinaus dermal wachsender Tumor der ekkrinen Schweißdrüsen. Bisher
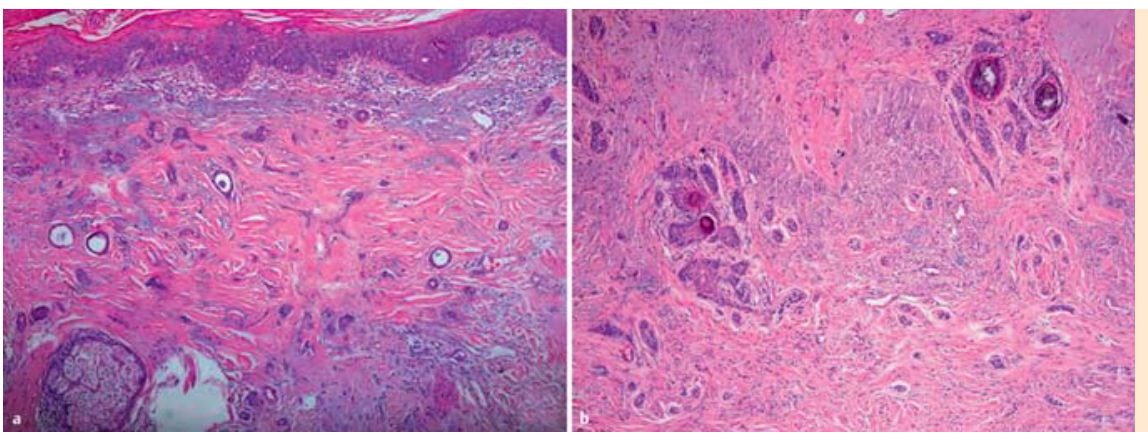

Abb. 2 a HE-Färbung, 200-fache Vergrößerung. Akanthotisch verbreiterte Epidermis mit Verlust der physiologischen Schichtung und zahlreichen Zell- und Kernpolymorphien. In den oberen Dermisabschnitten Keratinzysten und kleinere, teils syringoide Zellverbände mit blassem Zytoplasma und monomorphen Zellkernen. b HE-Färbung, 400-fache Vergrößerung. Kleine, teils tubuläre Epithelverbände mit vereinzeltem Nachweis von eosinophilem Sekret in hyalinisiert-fibrosiertem Stroma. 

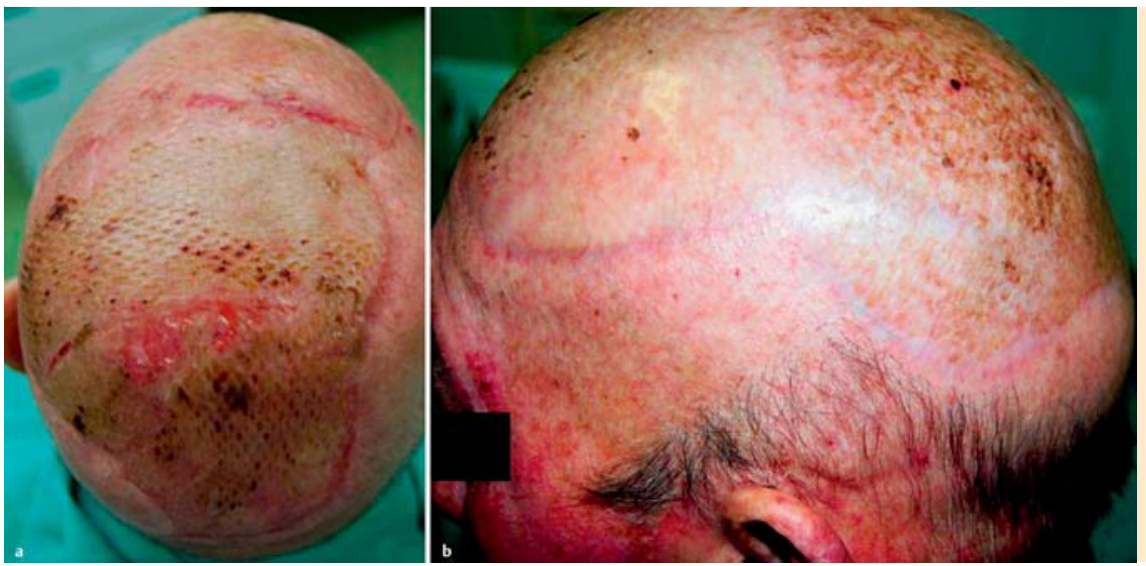

Abb. 4 Abschluss der operativen Maßnahmen 27 Tage (a) bzw. 6 Monate (b) nach Defektverschluss mittels Spalthauttransplantation.

wurden in der Literatur lediglich ca. 300 Fälle beschrieben [7]. Es wird ein diffus infiltrierendes Wachstumsmuster beobachtet. Die typische Lokalisation ist der zentrofaziale Bereich mit einem möglichen Überwiegen der linken Gesichtshälfte, das mediane Manifestationsalter liegt bei 65 Jahren (19-90 Jahre) [3]. Ausgehend von der bislang größten Fallsammlung von 48 Fällen besteht eine gewisse Bevorzugung des weiblichen Geschlechts (2:1) [3].

Histologisch ist das MAK durch einen zweischichtigen Aufbau charakterisiert. Das „benigne“ histologische Erscheinungsbild des Tumors in der oberen Dermis mit Hornzysten kann zu Fehldiagnosen, wie Trichoadenom, desmoplastisches Trichoadenom, Trichoepitheliom, aber auch Syringom Anlass geben. Erst die teils nestartig, überwiegend aber strangartig tubulär angeordneten Epithelverbände mit umgebendem hyalin-fibrosierten Stroma sowie das infiltrative Wachstumsmuster mit häufig vorzufindender Perineuralscheideninfiltration in den tieferen Abschnitten sind für die Diagnose MAK wegweisend. Meist sind keine Zellatypien nachweisbar. Daher ist zur Klärung der genannten Differenzialdiagnosen immer eine tiefe Exzisionsbiopsie unbedingt erforderlich.

Als Therapie der ersten Wahl wird die vollständige Exzision des Tumors mit mikrografisch kontrollierter Chirurgie empfohlen $[3,7]$. Hierbei konnte gezeigt werden, dass die endgültige Exzidatgröße einer großen Streuung unterliegt und im Median das 4-fache Areal der primären Exzision beträgt [3]. Ein Sicherheitsabstand bei primärer Exzision ist daher nicht definiert.

Zur Ätiopathogenese ist bisher wenig bekannt. Es wird angenommen, dass sich die Tumorzellen aus pluripotenten Keratinozyten mit der Fähigkeit zur adnexiellen Differenzierung entwickeln [7]. In einzelnen Studien wurde, wie bereits erwähnt, die Bevorzugung der linken Gesichtshälfte beschrieben. Hieraus wurde von einigen Autoren abgeleitet, dass der Tumor durch UV-Strahlen induziert werden könnte, da die linke Gesichtshälfte mehr der Sonne ausgesetzt sei (z.B. beim Autofahren) [3]. In einzelnen Fällen wurde auch die Entstehung von MAK in vorbestrahlten Gebieten beschrieben. Auch sehen einige Autoren einen möglichen Zusammenhang zwischen dem Auftreten des MAK und Immunsuppression [7].

Das Außergewöhnliche am vorliegenden Fall war einerseits die extreme Ausdehnung des Befundes vom Nacken bis in den Gesichtsbereich, die in dieser Form nur selten beschrieben wurde [4]. Zum anderen wurde sieben Jahre vor Diagnosestellung des MAK eine PDT bei histologisch nachweislicher Feldkanzerisierung des Capillitiums durchgeführt, damals ohne irgendeinen Hinweis auf ein MAK. Ein kausaler Zusammenhang zwischen dem Auftreten eines MAK und vorausgehender PDT, der hier nicht völlig ausgeschlossen werden kann, wurde bislang in der Literatur nicht beschrieben. Allerdings ist die kumulative UV-Exposition als potenzieller Faktor sowohl für die aktinische Schädigung als auch für die Induktion des MAK möglicherweise bedeutsam [7]. Andererseits lässt das Befundausmaß des MAK auf ein bereits jahrelanges Wachstum schließen, sodass der Einfluss der PDT fraglich bleibt.

Das Vorgehen bei inoperablem MAK, wie im vorliegenden Fall, ist nicht standardisiert. Zum Einsatz und zur Effektivität der Strahlentherapie liegen wenige und kontroverse Daten vor. In einem Fallbericht von Stein et al. wird nach einer anfänglichen Regression des Tumors 6 Monate nach Radiatio die Induktion einer aggressiveren Tumorentität beschrieben [8]. In einem weiteren Fallbericht wird die vollständige Abheilung eines MAK im Bereich der Unterlippe nach alleiniger Radiatio geschildert [9]. Zur adjuvanten Strahlentherapie mit mindestens $50 \mathrm{~Gy}$ und einer Sicherheitszone von $3-5 \mathrm{~cm}$, auch bei R1-Situation, berichtet eine aktuelle Publikation über eine Tumorkontrollrate von $98 \%$. Bei dieser retrospektiven Analyse von 14 Fällen wurden keine Transformationen mit aggressiverem Wachstum beobachtet [10].

Bei unserem Patienten besteht aktuell ein halbes Jahr nach Durchführung der Strahlentherapie Rezidivfreiheit. Das Auftreten von Rezidiven des MAK wurde jedoch noch nach bis zu 30 Jahren beschrieben [11], sodass wir eine klinische Nachsorge zumindest in halbjährlichen Abständen und Re-Biopsien im weiteren Verlauf vorsehen werden.

\section{Abstract}

\section{Bathing Cap-Like Microcystic Adnexal Carcinoma Associated with a Bowen Carcinoma after Photo- dynamic Therapy \\ $\nabla$}

Microcystic adnexal carcinoma (MAC) is a rare, often over years slowly growing tumor of the sweat glands with an aggressive infiltrative growth. It is histologically characterized by superficial epithelial nests with central keratin horn cysts. In the deeper regions syringoid epithelial nests with perineural growth are typical for this tumor entity.

We report on a 71-year-old male patient applied to our outpatient clinic with multiple carcinomata in situ on his head. Seven years ago he underwent a photodynamic therapy (PDT). Again we performed a PDT and excised a lesion on the occipital area of the head suspected as squamous cell carcinoma. After 
nine micrographically controlled excisions a defect resulted which involved the complete scalp. A R0 situation would only have been achievable with a destruction of the face. Therefore the wound was closed by mesh graft and a subsequent radiation therapy was started. Six months after radiation there is no histological evidence for a recurrence of the MAC.

This case report demonstrates that MAC can develop to an extreme dimension notwithstanding its clinical invisibility. Due to the rareness of the tumor only little is known about etiology and pathogenesis. An induction by UV exposure or ionising radiation is discussed. If PDT had an influence of the appearance of the MAC in this case, remains speculative.

\section{Literatur}

1 Goldstein DJ, Barr RJ, Santa Cruz DJ. Microcystic adnexal carcinoma: a distinct clinicopathologic entity. Cancer 1982; 50: 566-572

2 Hamsch C, Hartschuh W. Microcystic adnexal carcinoma - aggressive infiltrative tumor often with innocent clinical appearance. J Dtsch Dermatol Ges 2010; 8: 275-278
3 Chiller K, Passaro D, Scheuller $M$ et al. Microcystic adnexal carcinoma, forty-eigth cases, their treatment and their outcome. Arch Dermatol 2000; $136: 1355-1359$

4 Matsushita S, Uemura T, Imayama $S$ et al. Giant microcystic adnexal carcinoma of the scalp. J Dermatol 2008; 35: 726-728

5 Hoang MP, Dresser KA, Kapur P et al. Microcystic adnexal carcinoma: an immunohistochemical reappraisal. Mod Pathol 2008; 21: 178 - 185

6 Antley C, Carney M, Smoller B. Microcystic adnexal carcinoma arising in the setting of previous radiation therapy. J Cutan Pathol 1999; 26: 48 50

7 Wetter R, Goldstein G. Microcystic adnexal carcinoma: a diagnostic and therapeutic challenge. Dermatol Ther 2008; 21: 452 - 458

8 Stein J, Ormsby A, Esclamado R, Bailin P. The effect of radiation therapy on microcystic adnexal carcinoma: a case report. Head Neck 2003; 25 : $251-254$

9 Gulmen S, Pullon PA. Sweat gland carcinoma of the lips. Oral Surg Oral Med Oral Pathol 1976; 41: 643-649

10 Baxi S, Deb S, Weedon D et al. Microcystic adnexal carcinoma of the skin: the role of adjuvant radiotherapy. J Med Imaging Radiat Oncol 2010; 54: 477-482

11 Lupton G, Mcmarlin S. Microcystic adnexal carcinoma. Report of a case with 30-year follow-up. Arch Dermatol 1986; 122: 287-289 